\title{
On the Transmission of Double Watermarked Image over Rician FSO Channel
}

\author{
Bojan P. Prlincevic ${ }^{1}$, Stefan R. Panic ${ }^{2}$, Petar C. Spalevic ${ }^{3}$, Milan A. Misic ${ }^{1}$, Abdalmalik Amniesi ${ }^{4}$, \\ Vladimir Stanojevic ${ }^{4}$ \\ ${ }^{1}$ Higher Technical Professional School in Zvecan, \\ St. Nusiceva No.6,38227 Zvecan, Serbia \\ ${ }^{2}$ Faculty of Natural Science and Mathematics, University of Pristina, \\ K. Mitrovica, Serbia, \\ ${ }^{3}$ Faculty of Technical Sciences, University of Pristina, \\ Knjaza Milosa 7, K. Mitrovica, Serbia \\ ${ }^{4}$ Faculty of Electrical and Computer Engineering, Singidunum University, \\ Danijelova 32, Beograd, Serbia \\ b.prlincevic@vts-zvecan.edu.rs
}

\begin{abstract}
Performance analysis of double watermarked image transmission over the Rician turbulence FSO channel was carried out in this paper. First, we have considered the importance of copyright, robustness of watermark in FSO transmission and presented some algorithms for watermarking. Then, simulation of FSO transmission of double watermarked image over Rician channels has been carried out and obtained simulation results have been shown graphically. Quality of the extraction of the primary watermark and the secondary watermark was discussed in the function of FSO channel link parameter $K$ value.
\end{abstract}

Index Terms-FSO transmission; Rician channels; watermarking, image processing; discrete wavelet transforms.

\section{INTRODUCTION}

Exchanging of multimedia data (pictures, video, audio...) has become widespread with daily based usage of novel wireless technologies and rapid growth of digitization. In such environments copyright protection has risen as major issue. In such applications often some additional information (visible or invisible) incorporated into the transmitted data, is used with the aim of proving the ownership of the author, i.e. distributor, is very common in the practice. Inserting the hidden information into digital data for the purpose of proving the copyright is called a digital watermark [1]. Some basic characteristics of digital watermark are insensitivity, robustness, capacity, non-inverse and ability to provide indisputably positive proof of ownership [2]. For inserting the watermark in the data, many transformations could be used such as Discrete Cosine Transformation (DCT) [3], Discrete Wavelet Transformation (DWT) [4], Singular Value Decomposition (SVD transformation) [2], [5], and many other combinations of them are used for watermarking.

Development of transmission systems has led to the use of high-data-rate free-space optical (FSO) transmission systems. Contrary to radio frequency (RF) transmission,

Manuscript received 5 August, 2015; accepted 28 December, 2015.
FSO transmission possesses some properties (power concentration in a narrow beam, absence of wave interference, no need for free Fresnel zone obtainment, full duplex transmission possibility, small practical realization complexity, license-free transmission...) which favours the usage of FSO in many transmission scenarios. Also, short dimensions of wavelengths are used for FSO, which causes some other transmission advantages. Nevertheless, FSO transmission possesses some minor disadvantages, such are: acquisition and pointing are more complicated, attenuation can be caused by various atmospheric factors (i.e. turbulence, sun, fog, rain...).

Principal performance impairment that deteriorates link performance in FSO communications, especially for long distance transmissions is atmospheric turbulence-induced fading [6]. Temporal and spatial fluctuations of the laser beam that occur as a result of variations in the refractive index, caused by atmospheric turbulence and manifest as irradiance fluctuations in the received signals, are called FSO fading or scintillation. Very intensive FSO broadcasting of digital image and other data at high transfer speed (up to $10 \mathrm{~Gb} / \mathrm{s}$ ) for typical fade durations of few milliseconds, could cause inaccurate transmission or losing a large number of consecutive bits, which would result in higher bit-error rate (BER). Developing a number of FSO models in literature has been carried out due to the need of accurate prediction of the link performance under desired atmospheric conditions. Statistical distribution of atmospheric turbulence has been modelled with various models that provide excellent agreement between theoretical and experimental data, such are log-normal turbulence model, $K$ distribution model [7] and Gamma-Gamma model [8]. In [9] performances of image transmission over FSO channels, but without digital watermarking, were analysed.

In this paper, performance analysis of double watermarked image, transmitted over FSO transmission through Rician channel was carried out. In Section II the pseudocode of watermarking method and Rician FSO 
transmission has been presented. Obtained simulation results and performance analysis are presented in Section III Concluding remarks are given in Section IV.

\section{SYSTEM MODEL}

At the aperture plane of the receiver, received FSO signal is modeled as

$$
E_{r}(t, r)=u_{s}(t) \exp \left(j 2 \pi f_{c} t+\theta(t)\right) \exp [\chi(r)+j \phi(r)]
$$

where $r$ is the position vector on the receive aperture plane, $f_{c}$ is the optical carrier frequency, and $u_{s}(t) \exp (j \theta(t))$ denotes the complex envelope of the modulation signal. Here, $\chi(r)$ is the turbulence-induced amplitude fluctuations and $\phi(r)$ is the phase variations of the channel.

Assuming that the receiver integrates photocurrent for each bit period and removes bias due to background illumination [10], the photocurrent output is modelled as

$$
y_{T}(t)=x_{T}(t)+n_{T}(t),
$$

where $n_{T}(t)$ denotes Additive White Gaussian Noise (AWGN), has zero mean and variance of

$$
\sigma_{n}^{2}=\frac{B_{S} e^{2} \eta \pi}{2 h f_{c}} A^{2} D^{2},
$$

where $e$ denotes the electron's charge, $h$ is the Planck's constant, $\eta$ is the quantum efficiency of the photodetector, $B_{s}$ is the signal bandwidth, $D$ is the aperture diameter of receiver, and $A$ denotes the amplitude of Local Oscillator (LO) field at the demodulator.

Information carrying part of signal can be modeled as

$$
x_{T}(t)=\frac{e \eta \pi}{2 h f_{c}} A D^{2} u_{s}(t) \operatorname{Re}\left\{\alpha \exp \left(j 2 \pi f_{I F} t+\theta_{I F}(t)\right)\right\},
$$

where $f_{I F}=f_{c}-f_{L O}$ is the equivalent signal frequency, $f_{L O}$ is local oscillator frequency, $\theta_{I F}$ is the equivalent signal phase and $\alpha$ represents the effective FSO fading fluctuation of the channel. As shown in [11], [12], in order to model simultaneous effects of turbulence-induced amplitude fluctuations and phase aberrations, the Probability Density Function (PDF) of FSO fading amplitude will be modeled with Rician distribution, as

$$
f_{\alpha}(\alpha)=\frac{2 \alpha(1+K)}{\alpha^{2}} e^{-K-(1+K) \alpha^{2} / \overline{\alpha^{2}}} I_{0}\left(2 \alpha \sqrt{\frac{K(1+K)}{\alpha^{2}}}\right)
$$

where $I_{0}(x)$ is the zeroth-order modified Bessel function of the first kind [13], while parameter $K$, given by [12], stands for the ratio of the strength of the coherent component to the incoherent one in the detected field

$$
K=\left[\frac{\overline{\alpha^{2}}}{\sqrt{{\overline{\alpha_{r}}}^{4}+2{\overline{\alpha_{r}}}^{2}\left(\sigma_{i}^{2}-\sigma_{r}^{2}\right)-\left(\sigma_{i}^{2}-\sigma_{r}^{2}\right)^{2}}}-1\right],
$$

and

$$
\overline{\alpha^{2}}=\sigma_{i}^{2}+\sigma_{r}^{2}+{\overline{\alpha_{r}}}^{2},
$$

with parameters from (7), expressed in the terms of wavenumber, $k=2 \pi / \lambda$, the refractive index structure constant, and the propagation distance as in [12].

Finally, received instantaneous Signal-to-Noise Ratio (SNR) of the system after demodulation is given as

$$
\gamma=\frac{P_{x}}{\sigma_{n}^{2}}=\frac{\eta I_{S}}{h f_{c} B_{S}} \frac{\pi D^{2}}{4} \alpha^{2}
$$

where $I_{s}=\left|u_{s}(t)\right|^{2}$ is the average intensity of the optical field, and $P_{x}$ is the output signal power.

Algorithm for simulation of FSO transmission [14] of double watermarking image is accomplished in two phases consisting of the following steps:

Phase 1, Step 1: Form watermarked digital image $A^{*}(8-$ bit's) is created vector. Step 2: Obtained vector, with decimal elements, is translated to a vector with binary elements $B$ dimension $24 \times M \times N$. Step 3: On vector $B$ is applied Binary Phase Shift Keying (BPSK) modulation $x=2 \cdot B-1$. BPSK is modulation scheme that conveys data by changing the phase of a carrier wave by using two phases that are separated by $180^{\circ}$. This modulation is the most robust of all the PSKs since highest level of noise or distortion is necessary to make the demodulator reach an incorrect decision. Step 4: The BPSK modulated signal is transmitted trough the Rician turbulence channel: $y=h \times x+n$, with $h$ being vector that encompasses the influence of Rician fading, whose samples are generated according to (5), with respect to the parameter $K$, the ratio of the strength of the coherent component, defined as in (6). Vector $n$ encompasses the channel AWGN of defined SNR level. Step 5: Grey decoding with hard decisioning has been provided on $Y$, and vector $\bar{B}$ has been obtained. Step 6: Resulting watermarked digital image $\overline{A^{*}}$ is obtained from vector $\bar{B}$.

Phase 2, Step 1: From received data (watermarked 8-bit's image) $X_{1}$ is created vector $V$ with decimal elements. Step 2: Obtained vector is translated to vector with binary elements $B_{1}$ dimension $24 \times M \times N$. Step 3: On vector $V$ is applied Binary Phase Shift Keying (BPSK) modulation $B_{1}=2 \times V-1$. Binarysed vector $V$ is BPSK modulated and obtained vector $B_{1}$. Step 4: Vector $n$ encompasses the channel AWGN noise of the defined SNR level, and $\sigma$ component is obtained depending on the total power of Line-of-Sight (LOS) path and scattered pats. Step 5: Vector with the influence of Rician fading $h$, whose samples is generated according to (5), with respect to the parameter $K$, and the ratio of the strength of the coherent component defined in (6) is obtained as random component with following: $h=\sigma \times n$. Step 6: Gray decoding with hard decisioning has been provided on $Y: Y=h \times B_{1}+n$, and vector $\overline{Y_{1}}$ has been obtained. Step 7: Resulting watermarked digital image $\overline{X_{2}}$ vector is obtained from vector $\overline{Y_{1}}$.

Watermarking process papers consists of the following 
algorithms, according to the [15] and [16]:

Algorithm 1: inserting of the secondary watermark in the primary watermark. Algorithm 2: inserting of the primary watermark in the image. Algorithm 3: Extraction of the primary watermark from the image. Algorithm 4: Extraction of the secondary watermark from the image.

\section{1) Algorithm 1}

Algorithm of the insertion of the secondary watermark in the primary watermark is realized through the following steps:

Step 1: The first level of the DWT transformation is executed over the primary watermark $W_{P}$ size $(M / 2) \times(N / 2)$. Step 2: On the secondary watermark $W_{S}$ size $(M / 4) \times(N / 4)$, SVD transformation is applied. Step 3: SVD transformation is applied to all four subbands of the primary watermark $W_{P}{ }^{* k}=U_{W_{P}}^{k} \Sigma_{W_{P}}^{* k} V_{W_{P}}^{k T}$, where $k \in\{1,2,3,4\} \quad$ presents quadrants of DWT transformation of the primary watermark (LL, LH, HL and HH). Step 4: The singular values of all sub-quadrants of the primary watermark are modified with the singular values of the secondary watermark $\Sigma_{W_{P S}}^{k}=\Sigma_{W_{P}}^{k}+\alpha \times \Sigma_{W_{S}}$. Step 5: Inversion of SVD transformation is applied for obtaining the sub-quadrants. Step 6: The firs level of Inverse DWT transformation is applied for obtaining the primary watermark in which the watermark $W_{P S}$ is embedded.

2) Algorithm 2

Algorithm of the insertion of the primary watermark in the image is realized by the following steps:

Step 1: The first level of DWT transformation is applied to the original image $A$ size $M \times N$. Step 2: The Zig-zag sequence is applied to obtained subbands followed by construction of non-overlapping quadrants. Step 3: Over quadrants $A_{1}, A_{2}, A_{3}, A_{4}, A^{k}=U_{A}^{k} \Sigma_{A}^{k} V_{A}^{k T} \quad k \in\{1,2,3,4\}$ obtained in such way, SVD transformation is applied. Step 4: SVD transformation is applied to primary watermark $W_{P S}$, $W_{W_{P S}}=U_{W_{P S}} \Sigma_{W_{P S}} V_{W_{P S}}^{T}$. Step 5: Singular values of nonoverlapping rectangles are modified with singular values of the new primary watermark $\Sigma_{A_{P S}}^{k}=\Sigma_{A_{P}}^{k}+\alpha \cdot \Sigma_{W_{P S}}$. Step 6: Inverse SVD transformation is applied for obtaining of the subbands. Step 7: From the obtained non-overlapping rectangles a vector is constructed, over which the primary inverse zigzag transformation is applied to regain the nonoverlapping quadrants. Step 8: The inverse DWT transformation is applied to rectangles obtained in Step 7 to obtain an image with embedded watermark $A_{W}$.

\section{3) Algorithm 3}

Algorithm of the extraction of the primary watermark from the image is realized by the following steps:

Step 1: The first level of DWT transformation is applied to the watermarked image $A_{W}$ size $M \times N$. Step 2: The Zigzag sequence is applied to obtained subbands followed by construction of non-overlapping quadrants. Step 3: Over quadrants $A_{1}, A_{2}, A_{3}, A_{4}, A^{k}=U_{A}^{k} \Sigma_{A}^{k} V_{A}^{k T} \quad k \in\{1,2,3,4\}$ obtained in such way the SVD transformation is applied. Step 4: Singular values are extracted from the non- overlapping rectangles. Step 5: Inverse SVD transformation is applied to obtain sub-bands. Step 6: From the obtained non-overlapping rectangles a vector is constructed, over which the primary inverse zig-zag transformation is applied to regain the non-overlapping quadrants. Step 7: The Inverse DWT transformation is applied to rectangles obtained in Step 6 to obtain extracted primary watermark $W_{p e}$.

4) Algorithm 4

Algorithm of extraction of secondary watermark from the primary watermark is realized through the following steps:

Step 1: The first level of DWT transformation is applied to primary watermark $W_{p e}$. Step 2: SVD transformation is applied to sub-bands obtained from watermark $W_{P e}$. Step 3: The singular values of the secondary watermark are extracted from each quadrant. Step 4: The inverse SVD transformation is applied to obtain extracted secondary watermark $W_{S e}$.

\section{Simulation ANd Performance AnAlysis}

To simulate FSO transmission of watermarked images over the Rician turbulence fading channel following experiment is conducted: Step 1: Original image is watermarked with watermarking coefficient $\alpha_{1}=0.25$ (for primary watermark) and $\alpha_{2}=0.1$ (for secondary watermark). Step 2: On watermarked image $A^{*}$ is applied BPSK modulation. Step 3: BPSK modulated signal has been carried through Rician turbulence fading channel with AWGN. Step 4: Image has been reconstructed at the reception after performing Gray decoding with hard decisioning. Step 5: From reconstructed image watermark is extracted.

The imperceptibility of the watermark and quality of the transmitted image is quantified by Peak Signal-to-Noise Ratio (PSNR)

$$
P S N R=10 \log 10 \frac{2^{n}-1}{M S E},
$$

where MSE denote Mean Square Error between watermarked image and the transmitted image

$$
M S E=\frac{\sum_{i j}\left(x_{i j}-y_{i j}\right)^{2}}{M \times N},
$$

where $i=1 \ldots M, j=1 \ldots N$. The similarity measure between the original and transmitted image and watermark is evaluated by Normalized Correlation (NC) [5]

$$
N C=\frac{\sum_{i j}\left(x_{i j} y_{i j}\right)}{\sqrt{\sum_{i j}\left(x_{i j}\right)^{2}} \sqrt{\sum_{i j}\left(y_{i j}\right)^{2}}},
$$

where $i=1 \ldots M, j=1 \ldots N$ and BER

$$
B E R=\frac{\sum_{i j l}\left[\left(x_{i j}\right)_{l}\right]_{2} \oplus\left[\left(y_{i j}\right)_{l}\right]_{2}}{M \times N},
$$


where $i=1 \ldots M, j=1 . . N, l=1 . . n \quad x_{i j}$ is pixel of original image, $y_{i j}$ is pixel of restored image, $n$-number of bits, $M \times N$-the size of the image, and $\oplus$ denotes EXOR operator over each of $n$ pair of bits from $\mathrm{x}_{i j}$ and $\mathrm{y}_{i j}$.

In this experiment are used images presented on: Fig. 1. used as primary watermark (butterfly image, size $256 \times 256$ ), on Fig. 2 . used as secondary watermark (star image, size $128 \times 128)$, and images presented on Fig. 3. used as original images (Lena, Girl, Baboon and Peppers, size $512 \times 512$ ).

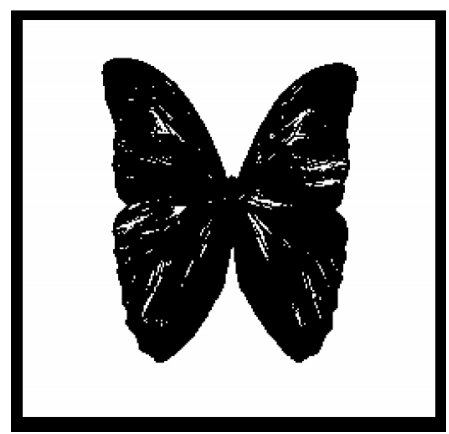

Fig. 1. Primary watermark image-Butterfly.

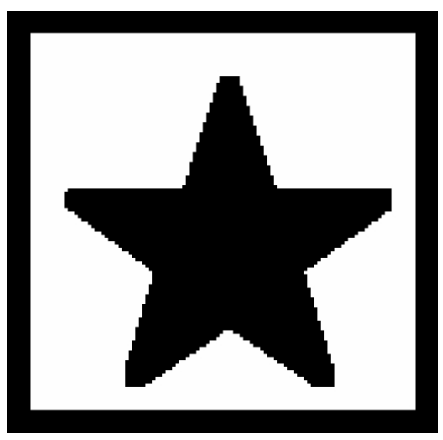

Fig. 2. Secondary watermark image-Star.

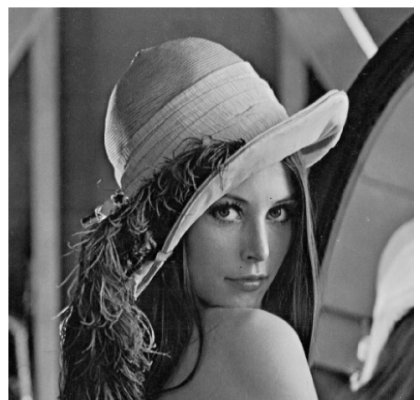

(a)

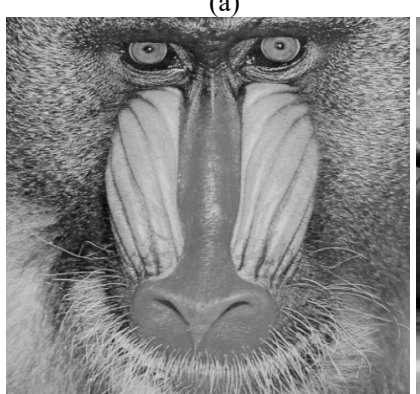

(c)

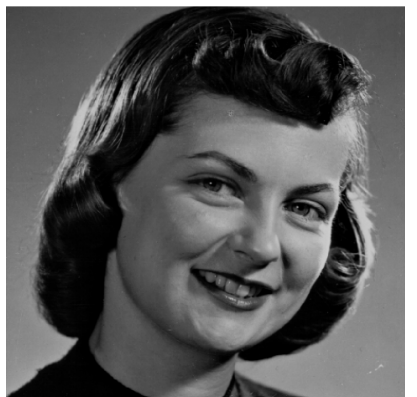

(b)

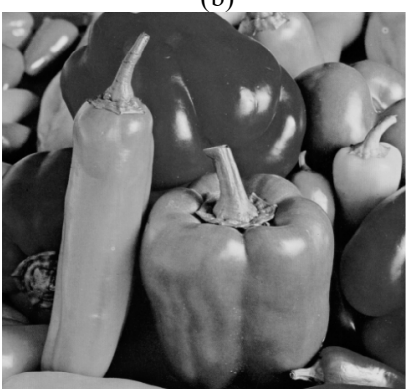

(d)
Fig. 3. Original images: a) Lena, b) Girl, c) Baboon and d) Pappers.

On Fig. 4 is presented quality measurement, PSNR for diferent transmitted images. On Fig. 5 is PSNR for extracted primary watermark from transmitted images on Fig. 6 is presented PSNR for extracted the secondary watermark from transmitted images.

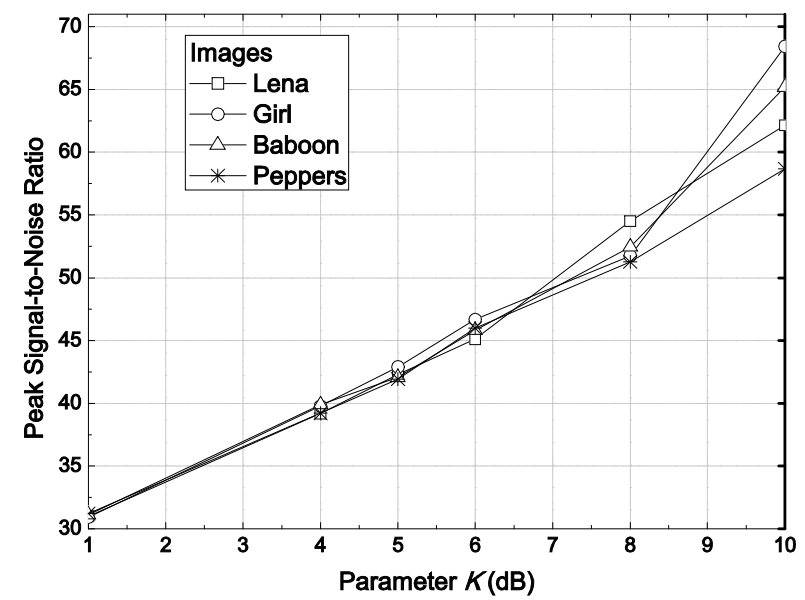

Fig. 4. PSNR for transmitted images: Lena, Girl, Baboon and Peppers.

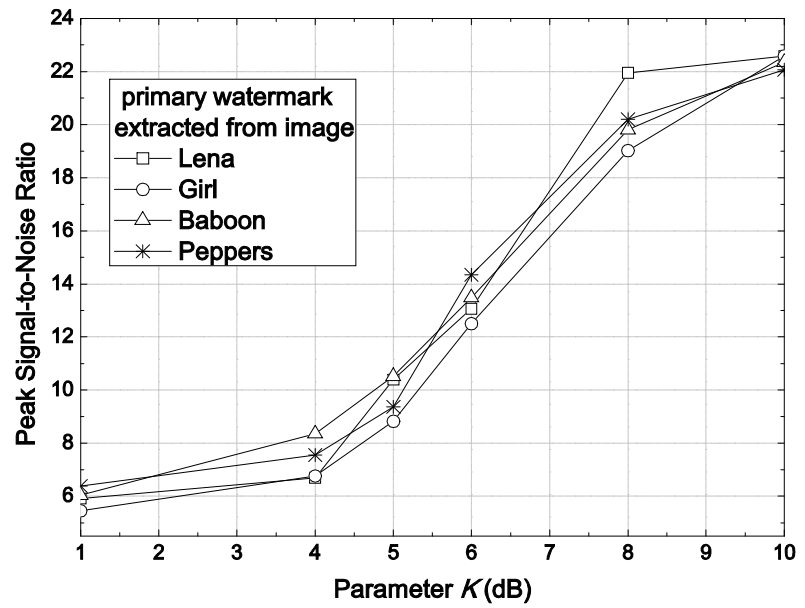

Fig. 5. PSNR for extracted primary watermark from transmitted images: Lena, Girl, Baboon and Peppers.

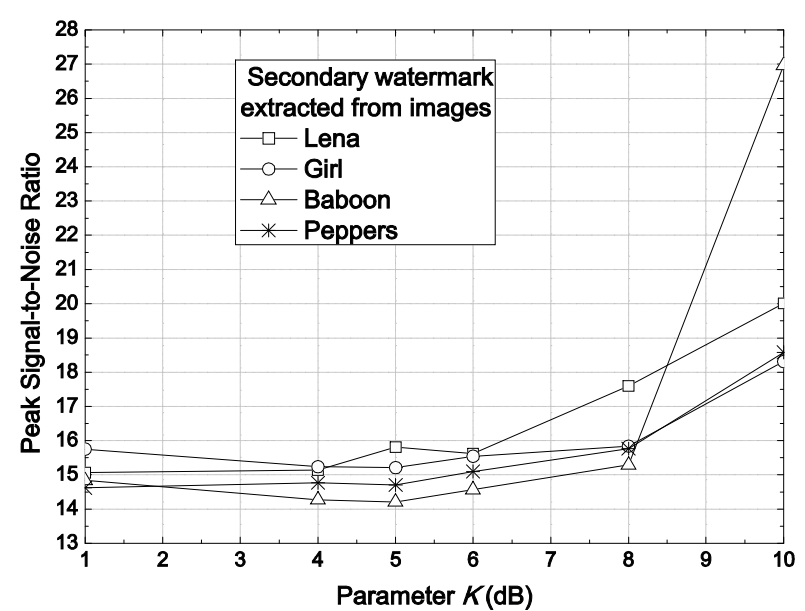

Fig. 6. PSNR for extracted secondary watermark from transmitted images Lena, Girl, Baboon and Peppers.

On Fig. 7 is presented quality measurement $\mathrm{NC}$ for different transmitted images, on Fig. 8. is presented NC for extracted primary watermark from transmitted images and on Fig. 9 is presented NC for extracted secondary watermark from transmitted images.

Analysing diagrams presented on Fig. 8 and on Fig. 9 one can be notice, that for parameter $K$ value $K>4$ primary 
watermark can be extracted with satisfied quality, secondary watermark is more robust and it can be extracted with satisfied quality with less value of parameter $K$.

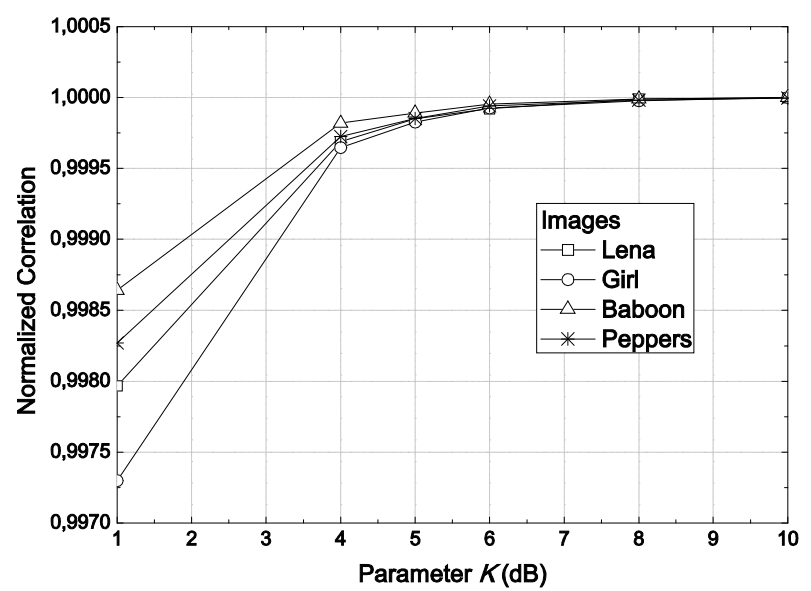

Fig. 7. Normalized correlation for transmitted images: Lena, Girl, Baboon and Peppers

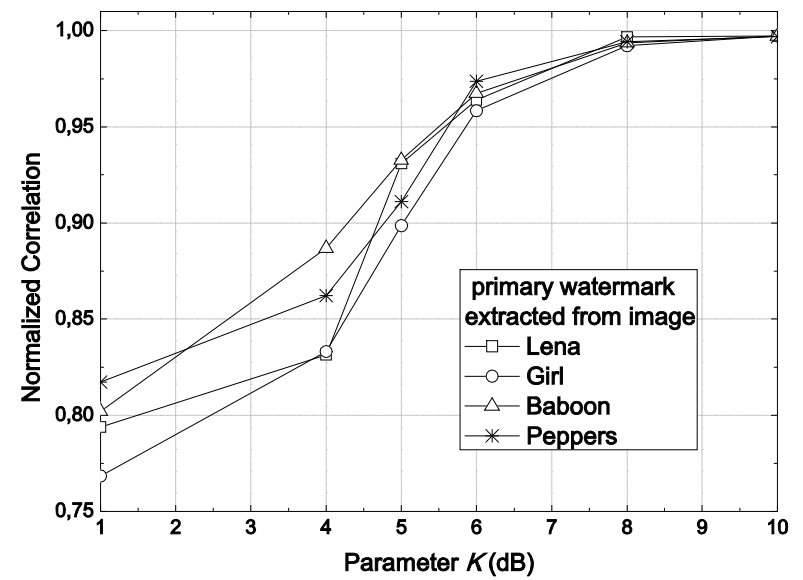

Fig. 8. Normalized correlation for extracted primary watermark, from transmitted images: Lena, Girl, Baboon and Peppers.

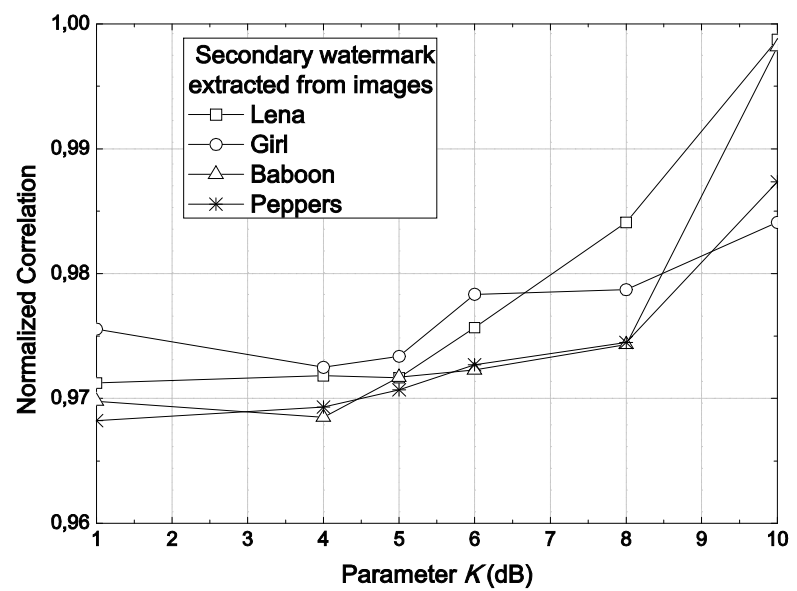

Fig. 9. Normalized correlation for extracted secondary watermark from transmitted images: Lena, Girl, Baboon and Peppers.

Quality measurement BER for transmitted images over the Rician turbulence fading channel with AWGN for different value of parameter $K$ are presented on Fig. 10 for image Lena, on Fig. 11 for image Girl, on Fig. 12 for image Baboon, and on Fig. 13 for image Peppers.

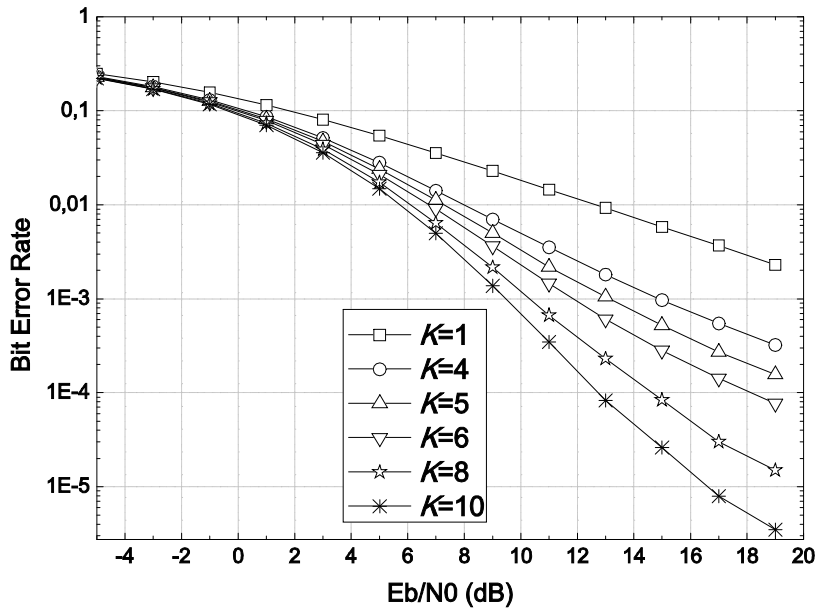

Fig. 10. BER for transmitted image Lena for different value of parameter K.

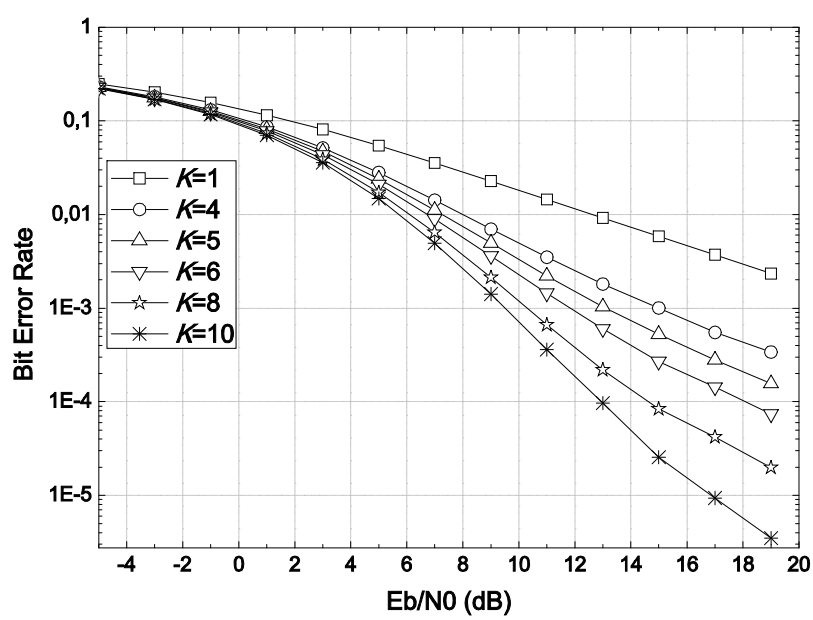

Fig. 11. BER for transmitted image Girl for different value of parameter $K$.

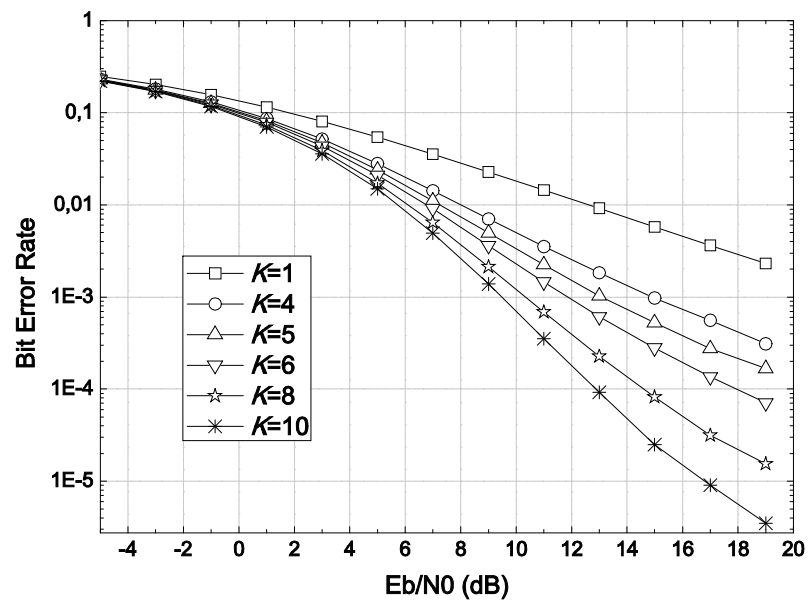

Fig. 12. BER for transmitted image Baboon for different value of parameter $K$.

As expected, FSO communication channels are prone to erroneous transmission, because influence of the weather conditions decreases transmission and image robustness. Analysing diagrams presented on Fig. 4-Fig. 13 one can notice, that for FSO channel parameter value $K=4$ quality measurements for transmitted image and extracted watermark reaches values that are satisfied, transmitted images are visibly undestroyed. 


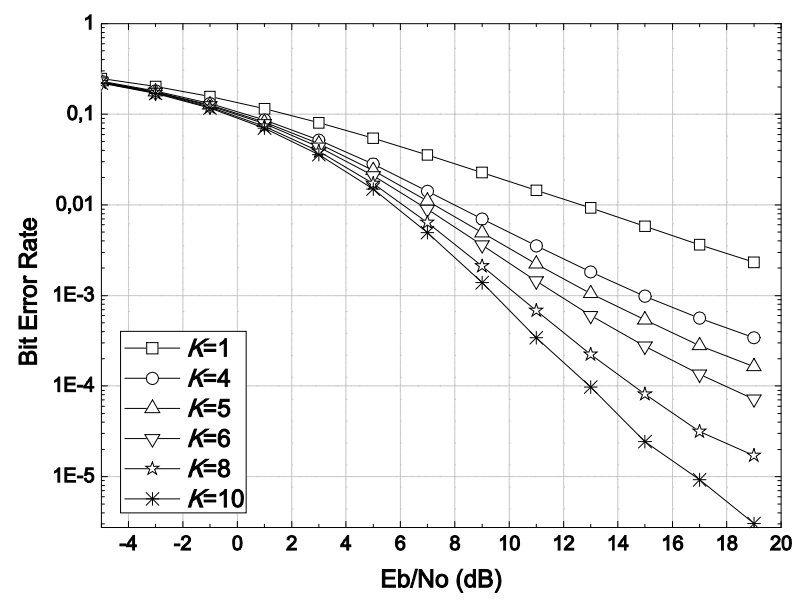

Fig. 13. BER for transmitted image Peppers for different value of parameter $K$.

\section{CONCLUSIONS}

We have analysed performances of last mile FSO transmission of the double watermarked image channel over the Rician turbulence channel. The purpose of paper was to provide theoretical analysis of low complexity FSO system with watermark image transmission, which could be implemented. Obtained values for standard performance measures at the reception: PSNR, NC, and BER, have been analysed as a function of FSO link parameters, in order to determine optimal system parameter values for obtaining necessary quality of service at the reception. It has been shown that for FSO channel parameter $K$ value $K>4$, quality measures for transmitted images and extracted primary and secondary watermarks reach satisfactory values of PSNR, NC $(N C>0.85)$ and BER. By applying analysis presented in this work, FSO link designers can estimate system parameters boundary values for achieving required values of PSNR, NC, and BER in similar last mile transmission scenarios of digital watermark image.

\section{ACKNOWLEDGMENT}

Authors thank the reviewers on their comments, which have helped us to improve the quality of the manuscript.

\section{REFERENCES}

[1] I J. Cox, M. L. Miller, J. A. Bloom, J. Fridrich, T. Kalker, Digital Watermarking and Steganography, Morgan Kaufmann Publishers, 2008.

[2] R. Liu, T. Tan, "A SVD based watermarking scheme for protecting rightful ownership", IEEE Trans. Multimedia, vol. 4, no. 1, pp. 121128, 2002. [Online]. Available: http://dx.doi.org/10.1109/ 6046.985560

[3] W. Chu, "DCT-based image watermarking using subsampling", IEEE Trans. Multimedia, vol. 5, no. 1, pp. 34-38, 2003. [Online]. Available: http://dx.doi.org/10.1109/TMM.2003.808816

[4] S. E. I. Baba, L. Z. Krikor, T. Arif, Z. Shaaban, "Watermarking of digital images in Frequency Domain", Int. Journal of Automation and Computing, vol. 7, no. 1, pp. 17-22, 2010. [Online]. Available: http://dx.doi.org/10.1007/s11633-010-0017-7

[5] C. C. Lai, "A digital watermarking scheme based on singular value decomposition and tiny genetic algorithm", Digital Signal Processing, vol. 21, no. 4, pp. 522-527, 2011. [Online]. Available: http://dx.doi.org/10.1016/j.dsp.2011.01.017

[6] X. Zhu, J. Kahn, "Free-space optical communication through atmospheric turbulence channels", IEEE Trans. on Communications, vol. 50, no. 8, pp. 1293-1300, 2002 [Online]. Available: http://dx.doi.org/10.1109/TCOMM.2002.800829

[7] H. Sandalidis, T. Tsiftsis, G. Karagiannidis, M. Uysal, "BER performance of FSO links over strong atmospheric turbulence channels with pointing errors", IEEE Comm. Letters, vol. 12, no. 1, pp. 44-46, 2008. [Online]. Available: http://dx.doi.org/10.1109/ LCOMM.2008.071408

[8] A. Al-Habash, L. C. Andrews, R. L. Phillips, "Mathematical model for the irradiance probability density function of a laser beam propagating through turbulent media", Optical Engineering, vol. 40, no. $8, \quad$ pp. 1554-1562, 2001. [Online]. Available: http://dx.doi.org/10.1117/1.1386641

[9] S. Zhao, B. Wang, Li Zhou, L. Gong, W. Cheng, Y. Sheng, B. Zheng, "Turbulence mitigation scheme for optical communications using orbital angular momentum multiplexing based on channel coding and wavefront correction", Physics. Optics, 2014. [Online]. Available: http://dx.doi.org/1401.7558

[10] A. A. Farid, S. Hranilovic, "Outage capacity optimization for free space optical links with pointing errors", Journal of Lightwave Technology, vol. 25, no. 7, pp. 1702-1710, 2007. [Online]. Available: http://dx.doi.org/10.1109/JLT.2007.899174

[11] A. Belmonte, J. M. Kahn, "Performance of synchronous optical receivers using atmospheric compensation techniques", Optics Express, vol. 16, no. 18, pp. 14151-14162, 2008. [Online]. Available: http://dx.doi.org/10.1364/OE.16.014151

[12] S. M. Aghajanzadeh, M. Uysal, "Diversity-multiplexing trade-off in coherent free-space optical systems with multiple receivers", Journal of optical Communications and Networking, vol. 2, no. 12, pp. 10871094, 2010. [Online]. Available: http://dx.doi.org/10.1364/JOCN. 2.001087

[13] I. S. Gradshteyn, I. M. Ryzhik, Table of Integrals, Series and Products. Academic Press, Elsvir, 2015.

[14] Z. Ghassemlooy, W. Popoola, S. Rajbhandari, Optical Wireless Communications, system and channel modelling with MATLAB, CRC press, Taylor\&Francis Group, 2013.

[15] B. Prlincevic, Z. Milivojevic, D Brodic, "Performance of DDS algorithm for insertain of double watermark", Int. scientific Conf. (UNITECH 2014), vol. 2, pp. 89-94.

[16] A. Mohammad, A. Alhaj, S. Shaltaf, "An improved SVD-based watermarking scheme for protecting rightful ownership", Signal Processing, vol. 88, no. 9 pp. 2158-2180, 2008. [Online]. Available: http://dx.doi.org/10.1016/j.sigpro.2008.02.015 